\title{
Numerical simulations of compact object binaries
}

\author{
Harald P. Pfeiffer \\ Canadian Institute for Theoretical Astrophysics, University of Toronto, \\ 60 St. George Street, Toronto, Ontario M5S 3H8, Canada
}

\begin{abstract}
Coalescing compact object binaries consisting of black holes and/or Neutron stars are a prime target for ground-based gravitational wave detectors. This article reviews the status of numerical simulations of these systems, with an emphasis on recent progress.
\end{abstract}




\section{Introduction}

Inspiraling and coalescing compact object binaries consisting of black holes and/or Neutron stars are of high importance to understand gravity and matter in extreme conditions. The strong gravitational fields require full nonlinear general relativity to describe them, and so compact object binaries allow exploration of curved space-time in the nonlinear and highly dynamic regime. The matter density inside Neutron stars is above nuclear density. Neutron stars therefore provide an avenue to study matter at super-nuclear densities, and compact object mergers allow such studies even in dynamic situations when the two objects collide.

Compact object binaries are therefore at the centre of attention for several scientific disciplines: The study of black hole binaries (BH-BH) sheds light on properties of general relativity in the genuinely nonlinear, dynamic regime (in a clean vacuum environment), ranging from black hole kicks (e.g. [1]) to the topological structure of event horizons [2-4]. Simulations of binaries involving one or two Neutron stars (BH-NS, NS-NS) [5,6] elucidate the connection between these systems and short gamma ray bursts. Finally, gravitational waves emitted by compact object binaries are the most promising source for gravitational wave detectors like Advanced LIGO [79], Advanced Virgo [10,11] and KAGRA [12]. Gravitational wave detectors require accurate waveform models and information about electromagnetic counterparts to optimally search for gravitational waves via matched filtering ("event detection"), and to extract the maximum amount of information about the source of gravitational waves, once such waves have been detected ("parameter estimation").

Direct numerical simulations of the full Einstein equations are an important cornerstone in the study of coalescing compact object binaries. These simulations have continued to progress swiftly, resulting in a large number of notable recent results. This article gives an overview of the current status of numerical simulations of compact object binaries. Our particular focus lies on recent advances, which have not yet been incorporated into longer or more specialized review articles (like $[6,13,14]$ ) or books (like [15]). Numerical simulations of higher-dimensional gravity (e.g. [16, 17]) and alternative theories of gravity (e.g. $[18,19]$ ) are advancing rapidly, but because of length limitations we shall restrict our attention to the "classical" compact object binaries (BH-BH, BH-NS, NS-NS) in four space-time dimensions in standard general relativity, the scenario of most direct relevance to gravitational wave detectors.

This article is organized as follows: Section 2 reviews BH-BH simulations, starting with numerical methods, recent advances, applications to gravitational wave science, and ending with $\mathrm{BH}-\mathrm{BH}$ simulations embedded in gaseous or electromagnetic environments. Section 3 deals with binaries with Neutron stars (BH-NS, NS-NS). We conclude in Sec. 4 with some thoughts about the future of the field.

\section{Black Hole-Black Hole Binaries}

\subsection{Numerical methods}

Since the numerical relativity breakthroughs in 2005 [20-22], several frameworks have emerged to simulate inspiraling and merging $\mathrm{BH}-\mathrm{BH}$ binaries. The major differentiating factors are the choices for the formulation of Einstein's equations (either the BSSNOK system [23-25] or the Generalized Harmonic (GH) system [20, 26-28]) and the choice of the numerical evolution algorithm, finite-differences (FD) or pseudo- 


\begin{tabular}{|c|c|c|}
\hline & BSSNOK+FD & GH+Spectral \\
\hline \multicolumn{3}{|c|}{ Initial data } \\
\hline Formulation & $\begin{array}{l}\text { Conformal method using } \\
\text { Bowen-York solutions [32-34] }\end{array}$ & $\begin{array}{l}\text { Conformal thin sandwich } \\
{[33,35]}\end{array}$ \\
\hline $\begin{array}{l}\text { Treatment of } \mathrm{BH} \\
\text { singularities }\end{array}$ & puncture data $[36]$ & $\begin{array}{l}\text { quasi-equilibrium } \\
\text { BH excision [37-39] }\end{array}$ \\
\hline Num. algorithm & $\begin{array}{l}\text { pseudo-spectral [40] } \\
\end{array}$ & pseudo-spectral [41] \\
\hline $\begin{array}{l}\text { Low-eccentricity } \\
\text { orbital param's }\end{array}$ & post-Newtonian inspiral [42] & $\begin{array}{l}\text { iterative eccentricity removal } \\
{[43,44]}\end{array}$ \\
\hline \multicolumn{3}{|c|}{ Evolution } \\
\hline Formulation & BSSNOK $[23-25]$ & $\begin{array}{l}\text { Generalized harmonic \& con- } \\
\text { straint damping }[20,26-28]\end{array}$ \\
\hline Gauge conditions & $\begin{array}{l}\text { evolved lapse and shift } \\
{[45-47]}\end{array}$ & $\begin{array}{l}\text { Harmonic }\left(H^{\mu}=0\right) \\
\text { and/or evolved } H^{\mu}\end{array}$ \\
\hline $\begin{array}{l}\text { Treatment of } \mathrm{BH} \\
\text { singularities }\end{array}$ & moving punctures $[21,22]$ & BH excision [49] \\
\hline $\begin{array}{l}\text { Treatment of } \\
\text { outer boundary }\end{array}$ & Sommerfeld BC & $\begin{array}{l}\text { minimally-reflective, } \\
\text { constraint-preserving }[28,50]\end{array}$ \\
\hline Discretization & $\begin{array}{l}\text { high-order finite-differences } \\
{[51,52]}\end{array}$ & pseudo-spectral methods \\
\hline Mesh-refinement & adaptive mesh refinement & domain decomposition $[41,48]$ \\
\hline \multicolumn{3}{|c|}{ Diagnostics } \\
\hline $\mathrm{BH}$ & \multicolumn{2}{|c|}{ Apparent horizon finder, quasi-local spin measures } \\
\hline GW extraction & \multicolumn{2}{|c|}{ Newman Penrose scalars, Regge-Wheeler-Zerilli } \\
\hline \multicolumn{3}{|c|}{ Major codes } \\
\hline Infrastructure & $\begin{array}{l}\text { BAM, Cactus, Einstein } \\
\text { Toolkit, Hahndol }\end{array}$ & SpEC \\
\hline Codes & $\begin{array}{l}\text { BAM, Hahndol, LazEv, Lean, } \\
\text { Llama, MayaKranc, UIUC }\end{array}$ & SpEC \\
\hline
\end{tabular}

Table 1. Ingredients into a $\mathrm{BH}-\mathrm{BH}$ simulation, and the most common choices for the two major frameworks in use to solve $\mathrm{BH}-\mathrm{BH}$ spacetimes.

spectral methods. Pretorius' first simulations employed the combination GH+FD $[20$, 27], and this code has since been applied to a variety of physical scenarios including high energy collisions of $\mathrm{BH}-\mathrm{BH}[29]$, eccentric $\mathrm{BH}-\mathrm{BH}[30]$ and studies of gravity in different space-time dimensions $[17,31]$.

However, in regard to the main focus of this section, $\mathrm{BH}-\mathrm{BH}$ simulations of direct relevance to gravitational wave detectors, the combinations BSSNOK+FD and $\mathrm{GH}+$ Spectral have emerged as the leading frameworks, and so we shall describe these in some detail. The BSSNOK+FD framework is built around the BSSNOK [23-25] evolution equations coupled with finite-difference (FD) approximation schemes. The GH+Spectral framework utilizes the generalized harmonic $(\mathrm{GH})$ equations [20, 2628 ] and multi-domain spectral methods. These frameworks differ in a large number of the individual elements that are required to compute a gravitational waveform with numerical relativity. Table 1 lists the main choices that are made within the two frameworks for setting initial data, performing the evolution and analyzing the 
resulting data. Besides formulation and numerical algorithm used for the evolution equations, these choices include formulation and numerical methods for the initial data, diagnostics tools, and factors that determine the physics of the scenario under consideration (e.g. low orbital eccentricity). While Table 1 conveys the broad picture, it must necessarily simplify and omit details. For instance, Pretorius' breakthrough work [20] does not fit into these categoriesł.

The choices shown in Table 1 are interrelated and only certain combinations are feasible. In particular, one cannot simply exchange specific elements between BSSNOK+FD and GH+Spectral. Let us illustrate some of these dependencies: Moving puncture evolutions performed in the BSSNOK+FD framework require very specific gauge conditions (Gamma-driver and 1+log slicing [45-47]) to keep the punctures stable during the evolution. Because the BH's are not excised, moving puncture evolutions require initial data covering the entire initial-data hypersurface. Therefore, a moving puncture evolution cannot be started from the excision initialdata used for the spectral evolutions§.

Puncture data covers the entire initial-data hypersurface, so it seems that $\mathrm{GH}+$ Spectral evolutions could use puncture data. However, this is also non-trivial: Singularity excision requires careful control over the location and shape of the excision boundary $[49,56]$. This is more difficult when the black hole horizons change on short time-scales, in particular during merger $\|$ and early in an evolution when the coordinate location of the black holes and the gauge settles down. Conformal thin sandwich initial data (as used in the GH+Spectral approach) reduces significantly such transients and is therefore more suitable for $\mathrm{GH}+$ Spectral than puncture initial data.

Trumpet initial data reduces initial transient apparent horizon motion [57] compared to standard puncture initial data, however, so far trumpet initial data not been used regularly for production $\mathrm{BH}-\mathrm{BH}$ simulations.

To summarize some key properties of the two BH-BH frameworks:

- BSSNOK+FD simulations are generally considered more robust and "easier" because of the lack of black hole excision and the larger expertise of how to stabilize finite-difference methods. Moreover, code-infrastructure [58-60], including adaptive-mesh-refinement (Carpet [61,62]) and apparent horizon finders [63], are publicly available. Several independent research groups use these tools and have obtained a tremendous wealth of results (e.g. black hole kicks). The major codes are listed in Table 1 and more details are available in the related proceeding contributions describing the NINJA collaboration [64].

- Spectral methods, in contrast, require BH excision and are very sensitive to the presence of ill-defined elements in the problem (e.g. outer boundary conditions, handling of inter-domain boundaries where grids of different resolution touch, or filtering). Significant care and expertise is needed to analyze and control all relevant aspects of the evolution, and only one spectral BH-BH code exists: The Spectral Einstein Code SpEC [65], developed by the SXS collaboration between Cornell, Caltech, CITA and Washington State University.

$\ddagger$ Pretorius used the generalized harmonic evolution system with an adaptive-mesh-refinement finitedifference code, starting from initial data that contained nonsingular balls of scalar field that collapsed to BH's early in the evolution.

$\S$ Proposals to fill-in the BH interiors of excision initial-data [53-55] have not been systematically pursued.

\| Control of the excision boundary was one of the major obstacles in obtaining BH-BH mergers with GH+Spectral, cf. $[49,56]$. 
- The key advantage of spectral methods is their fast (exponential) convergence. Therefore, high accuracy can be obtained with comparatively low computational cost. The high accuracy/lower cost permeates the entire "simulation pipeline": Lower numerical noise (due to higher accuracy) makes it easier to perform certain fits that are required in iterative eccentricity removal (see Sec. 2.2.2); the low computational cost makes it possible to perform longer simulations; the clean waveforms extracted at finite radius are more amenable to extrapolation to obtain the asymptotic waveform at infinitely large distance; high accuracy also makes it easier to compute and analyze quantities that depend on higher derivatives of the evolved fields, for instance black hole vortices and tendices [66,67].

- To date, many more simulations have been performed with BSSNOK+FD than with $\mathrm{GH}+$ Spectral, but the $\mathrm{GH}+$ Spectral simulations are longer and more accurate. Both techniques have found their place, depending on the precise needs of the science to be studied. However, this conventional wisdom slowly changes, as the BSSN+FD simulations become more accurate and achieve longer simulations, whereas the GH+Spectral simulations become more robust and the number of GH+Spectral simulations increases [64].

While simulations work successfully, we caution the reader that both frameworks utilize heuristically determined ingredients: Gauge conditions and constraint damping terms contain parameters chosen by trial and error, and mesh structures are tuned based on user experience. Therefore, parameter choices working in one region of parameter space may require adjustments for other regions (e.g. [68-70]). Furthermore, constraint damping parameters impact the accuracy and quality of the simulation beyond their main purpose of preserving the constraints [71]. The presence of user-tuned parameters has further implications: On the negative side, it is not guaranteed that the current techniques work in unexplored regions of parameter space. On the positive side, it is conceivable - even likely- that further tuning of code parameters will enhance accuracy and efficiency of future simulations compared to todays runs.

\subsection{Recent advances}

Given the rather mature state of $\mathrm{BH}-\mathrm{BH}$ simulations, recent advances are incremental, pushing and refining capabilities, and confirming assumptions that were made in previous years to achieve fast progress.

2.2.1. New records Let us start with some "new records": With increasing massratio $q=M_{1} / M_{2} \geq 1$, fully numerical simulations become more challenging because the computational cost increases (the small black hole must be resolved), and because experience of code tuning from comparable mass binaries is no longer applicable. Two groups pushed the mass-ratio of BH-BH simulations up to $q=100$ : Sperhake et al [72] examine head-on collisions of a small black hole with a large black hole. Lousto and Zlochower [73] perform a numerical simulation of the last two orbits of a BH-BH binary of mass-ratio $q=100$. Both these calculations are very impressive, requiring not only a large amount of CPU resources, but also improvements and tuning of the computational infrastructure (e.g. location of mesh-refinement regions). The RIT group [74-76] extracts $\mathrm{BH}$-trajectories from numerical simulations of mass-ratio up to $q=100$. These trajectories are then used in perturbative calculations to compute the waveforms of high mass-ratio binaries. 
Turning to spin, the long-standing bound

$$
\frac{S}{M^{2}} \equiv \chi \lesssim 0.93
$$

was recently broken. Certain black hole properties vary significantly between $\chi=0.93$ and $\chi=1$ and therefore angular momentum may not be the most useful measure of extremality. For instance, the rotational energy of a black hole with $\chi=0.93$ is only $59 \%$ of the rotational energy of a maximally rotating black hole. When taking rotational energy as the metric, the bound (1) is far from extremality.

Equation (1) represents the maximal spin that is achievable with puncture initial data [77], and therefore, all $\mathrm{BH}-\mathrm{BH}$ simulations within the BSSN-OK framework are limited by this bound. BH spins higher than the bound (1) are achieved with the more general initial-data formalism utilized within the GH+Spectral framework. Lovelace et al [78] construct initial data and perform short evolutions of BH-BH binaries with spins of 0.97 . More recently, Lovelace et al $[56,79]$ compute complete inspiral/merger/ringdown simulations for equal mass $\mathrm{BH}-\mathrm{BH}$ binaries with aligned spins of 0.97 and anti-aligned spins of 0.95 . These simulations require delicate control of the excision boundaries and demonstrate that the SpEC code has significantly matured.

The $\chi=0.97$ aligned spin simulation [56] also carries another new record: It is presently the longest published complete $\mathrm{BH}-\mathrm{BH}$ simulation, lasting about 25 orbits before merger and ringdown, for an evolution time of $7000 \mathrm{M}$. The longest incomplete simulation (i.e. inspiral only) so far is presented by Le Tiec et al. [80], lasting 34 orbits and 11,000M. All these extremely long simulations were obtained with SpEC. The simulations presented in Ref. [80] range up to mass-ratio 8, all in excess of 20 orbits, and are used to compare periastron advance in $\mathrm{BH}-\mathrm{BH}$ binaries with several analytical approximation schemes.

The last year has also witnessed another increase in the largest known black hole kick from inspiralling $\mathrm{BH}-\mathrm{BH}$. To remind the readers, in 2007 the "BH super-kick configuration" was discovered [82-84], with BH kicks close to $4000 \mathrm{~km} / \mathrm{sec}$. In 2011, Lousto and Zlochower [1] demonstrated that tilting the BH spins from the superkick configuration toward partial alignment with the orbital angular momentum can increase the $\mathrm{BH}$ kicks to almost $5000 \mathrm{~km} / \mathrm{sec}$. Partial alignment of the $\mathrm{BH}$ spins with the orbital angular momentum allows the BH's to spiral closer to each other, where the higher velocities enhance the BH kick.

2.2.2. Improved techniques Eccentricity removal has been extended to precessing binaries: Isolated $\mathrm{BH}-\mathrm{BH}$ binaries originating from binary stars are expected to have vanishing orbital eccentricity when they enter LIGO's frequency band $[85,86]$. To achieve low eccentricity in a numerical simulation requires careful choices for orbital frequency $\Omega_{0}$ and radial velocity $v_{r}$ of the individual black holes (or, equivalently, tangential and radial linear momentum of the BH's). These parameters can be chosen based on post-Newtonian information, either in closed form or by integrating post-Newtonian ordinary differential equations for two point-masses starting at large separation. The post-Newtonian coordinates and velocities are then used in the construction of BH-BH initial-data. This approach $[42,87]$ is computationally inexpensive and achieves eccentricities of a few 0.001 for low-spin and comparable

ฯ Interactions of high-velocity black holes result in even larger kicks [81]. 
mass BH-BH, and somewhat higher eccentricity for high spins and unequal mass BH$\mathrm{BH}$.

One can also adjust orbital parameters iteratively [43,88]: One begins with a reasonable first guess for $\Omega_{0}$ and $v_{r}$ (perhaps based on post-Newtonian information), evolves for about two orbits, analyzes the orbital trajectories, and then adjusts $\Omega_{0}$ and $v_{r}$. With the adjusted initial orbital parameters, one constructs a new $\mathrm{BH}-\mathrm{BH}$ initial data set, and performs a new evolution. This technique is computationally more expensive but achieves eccentricities as small as $\sim 10^{-5}$. Recently, Buonnano et al [44] extended this technique to precessing $\mathrm{BH}-\mathrm{BH}$ systems. Ref. [44] performs a postNewtonian analysis demonstrating that spin impacts iterative eccentricity removal only for eccentricities $10^{-4} \ldots 10^{-3}$ (the exact bound depends on the BH-spins and the initial black hole separation). Furthermore, Ref. [44] demonstrates that $e \sim 10^{-4}$ can indeed be reached for several BH-BH configurations with dimensionless spins of 0.5. This work uses the GH+Spectral approach with the SpEC code. Tichy et al [89] proposed an alternative iterative technique within the BSSNOK+FD framework and reach eccentricities of a few $\times 10^{-3}$. Very recently Pürrer et al. [90] study iterative eccentricity removal for moving puncture simulations based on the gravitational waveforms.

Cauchy Characteristic extraction (CCE) [91-94] extracts certain data from a standard $3+1$ evolution code (as described in Sec. 2.1) and evolves these data with a separate characteristic code to future null infinity. At future null infinity, gravitational radiation is unambiguously defined and so this technique promises gauge invariant waveforms, improving on the more widely used technique to extrapolate finite-radius waveforms to infinite extraction radius [95]. In recent papers, Babiuc et al $[93,94]$ improve the PITT CCE code, and perform careful convergence tests. This code is publicly available as part of the Einstein Toolkit $[60,96]$. With the recent improvements and public availability of the CCE code, I expect rapid increase in the use of this post-processing tool. While CCE is gauge-invariant, it requires initialization at the begin of the numerical simulation. Bishop et al [97] investigate the impact of different initialization strategies and found some impact onto the CCE waveform. Furthermore, CCE cannot remove errors that were introduced in the underlying $3+1$ numerical evolution. For instance, if the outer boundary conditions of the $3+1$ evolution admit unphysical incoming radiation (or reflected outgoing radiation), then such radiation will appear in the CCE waveform.

2.2.3. Code validation and consistency checks Several papers confirmed that BH-BH simulations indeed work as expected thus further validating the numerical techniques:

Owen $[98,99]$ (extending work by Campanelli et al [100]) analyzes carefully a SpEC simulation of an equal-mass, non-spinning BH-BH. He confirmed with a multipolar analysis that the remnant black hole settles down to Kerr with the correct quasi-normal mode falloffs. The combination of gauge-invariant quantitites with the high accuracy of the SpEC code allows Owen to confirm agreement to many significant digits, usually to better than 1 part in $10^{5}$ and sometimes to better than 1 part in $10^{9}$. Refs. $[98,100]$ also confirm that the space-time of a black hole merger approaches Petrov type $\mathrm{D}$ at late times after the $\mathrm{BH}-\mathrm{BH}$ merger.

Hinder et al [101] perform a careful analysis of the asymptotic fall-off of the Newman Penrose scalars, revisiting [102]. The fall-off rates agree with expectations,

$$
\Psi_{n} \sim \frac{1}{r^{5-n}},
$$


where $n=0, \ldots 4$ labels the individual Newman-Penrose scalars, $\Psi_{0}, \ldots, \Psi_{4}$.

The NINJA-2 project [103] collected about 40 numerical waveforms, with some configuration contributed multiple times by different codes. These duplicate waveforms allow consistency checks between waveforms computed independently by different codes. Overlap calculations between $(2,2)$ modes of hybridized waveforms show disagreements broadly in line with the expected errors of the different numerical codes and hybridization procedures. Comparisons of higher order modes have not yet been performed and are planned for future work. For details, see the separate NINJA-2 contribution to the Amaldi proceedings [64].

2.2.4. Future improvements Several recent directions of research may have an impact on accuracy and efficiency of $\mathrm{BH}-\mathrm{BH}$ simulations in the future: The conformal Z4-system [104] combines features from BSSNOK with the constraint damping of the generalized harmonic equations, resulting in improved suppression of constraint violations (compared to BSSNOK). Witek et al [105] developed a generalized BSSNOK system, which encompases BSSNOK as a special case. They find that their extension improves numerical behavior of BSSN. Existing BSSNOK codes can easily be adopted to either the conformal Z4 system or the generalized BSSNOK system. Bona et al [106] presented a Lagrangian for the Z4 system, which facilitates the use of symplectic integrators for Einstein's equations.

Improved efficiency or reduced wall-clock run-time are in dire need, given that a high-quality $\mathrm{BH}-\mathrm{BH}$ simulation requires months to complete. At least two approaches are under development: Several groups [96,107,108] work on porting numerical relativity codes to graphical-processing-units (GPUs), usually within the CUDA framework, opening up the possibility that in the future GPUs may accelerate BH-BH simulations by a significant factor. Lau et al [109,110] explore novel time-stepping algorithms which circumvent the Courant timestep limit, and promise the ability to take significantly larger timesteps than current codes (see also [111]).

\section{3. $B H-B H$ waveforms for gravitational wave astronomy}

The primary motivation for the intense activity in $\mathrm{BH}-\mathrm{BH}$ research lies in gravitational wave astronomy. Gravitational wave detectors require reasonably accurate waveform templates to detect GWs, and more accurate waveforms to extract detailed knowledge about the source properties (parameter estimation). As discussed in detail in the review [112], one first performs $\mathrm{BH}-\mathrm{BH}$ simulations at discrete points in parameter space. One then constructs analytical waveform models (continuous in parameters) from these $\mathrm{BH}-\mathrm{BH}$ simulations, which are used for $\mathrm{GW}$ data-analysis purposes. This sequence of steps has been carried out several times [113-120], based on different numerical simulations, different types of analytical waveform models, and for different regions of parameter space (again, we refer to Ref. [112] for details).

The NINJA projects $[64,103,121]$ give a good sense of the rate of progress of $\mathrm{BH}-\mathrm{BH}$ simulations for GW-modeling. The initial NINJA project [121] in 2008 consisted of about 20 numerical relativity simulations lasting on average about $12 \mathrm{GW}$ cycles before merger, without any length- and accuracy-requirements. The current NINJA-2 project $[64,103]$ collected about 40 waveforms with on average about 20 GW cycles. NINJA-2 also expands the coverage of parameter space by including more spinning waveforms, the simulations are more accurate, and one carefully attaches post-Newtonian inspirals to the numerical waveforms. The second major ongoing 
effort, the NR-AR collaboration [122] is assembling a yet larger number of waveforms of average length of about $30 \mathrm{GW}$ cycles, and with yet higher accuracy than NINJA-2. Each one of these efforts takes about two years to complete, demonstrating the high complexity of computing, validating, and collecting high-quality $\mathrm{BH}-\mathrm{BH}$ waveforms.

One obstacle toward computation of high-quality waveforms in a shortage of experienced researchers capable of running $\mathrm{BH}-\mathrm{BH}$ codes and capable of improving the efficiency, robustness and automation of the codes. Furthermore, there were unexpected difficulties in scaling BSSN-OK simulations to greater length and higher accuracy and for the SpEC code to obtain robust and automatic mergers. Finally, computational resources are also constrained: As a rule of thumb, for "easy" parameter choices (moderate spins $\sim 0.5$, moderate mass-ratios $\sim 2$, moderate length, $\sim 10$ orbits), a single state-of-the-art BH-BH simulation requires on the order of 100,000 CPU-hours ${ }^{+}$. This CPU-cost is of course dependent on the length $T / M$ of the simulation (measured in units of the total mass $M$ ). For given symmetric massratio $\nu$, initial orbital frequency $\Omega_{i}$ or number $N$ of orbits to merger, lowest order post-Newtonian expressions [123] yield:

$$
\begin{aligned}
\frac{T}{M} & \approx \frac{5}{256} \nu^{-1}\left(M \Omega_{i}\right)^{-8 / 3}, \\
\frac{T}{M} & \approx 5 \nu^{3 / 5}(2 \pi N)^{8 / 5} .
\end{aligned}
$$

Halving the initial orbital frequency $\Omega_{i}$ or doubling the number of orbits $N$ increases $T$ by a factor $\sim 6$ or $\sim 3$, respectively. The increase in CPU-cost is even higher, to preserve phase-accuracy over the longer inspiral. Higher mass-ratio and higher spins increase the CPU-cost further.

Equations (3) and (4) basically force a trade-off between the length of each simulation and the number of simulations that can be performed with limited CPU resources. Reasonable accuracy for event-detection can be achieved with $\sim 10$ orbits $[124,125]$, however, optimal parameter extraction requires numerical simulations starting at much lower initial frequency [126-128]. The low starting frequency is necessary because the accuracy of the hybrid waveform is primarily limited by the errors of the 3.5-th order post-Newtonian waveforms that are attached before the start of the numerical simulation.

2.3.1. Exploration of parameter space The entire parameter space for $\mathrm{BH}-\mathrm{BH}$ binaries is nine-dimensional: mass-ratio, two spin-vectors, and two parameters related to eccentricity (eccentricity and phase at periastron). Essentially all efforts to explore this parameter space so far have focused on non-eccentric binaries, and even for noneccentric binaries, only the following low-dimensional subspaces have been covered in detail (see the NINJA-2 contribution [64] for details and references):

- Non-spinning binaries with mass-ratio $1 \leq q \leq 10$.

- Equal mass binaries with equal spins parallel to the orbital angular momentum.

- Circular, non-precessing binaries form a three dimensional parameter space (massratio and spin-magnitudes of the two spins parallel with the orbital angular momentum). This space has not been covered extensively yet, with most efforts having been focused on the two one-dimensional subspaces just mentioned.

+ The SpEC code is more efficient than the BSSN-OK codes. But SpEC-simulations focus on longer and more accurate simulations, resulting in a CPU cost of the same order of magnitude. 
The vast seven-dimensional space of precessing binaries on circular orbits has received so far surprisingly little attention: Campanelli et al [129] compute one waveform with mass-ratio $q=1.25$, spins of 0.6 and 0.4 , lasting about nine orbits. A few configurations are used to demonstrate BH-BH mergers in Szilagyi et al. [48], but without discussion of gravitational waveforms. Ref. [44] perform several inspiral simulations of precessing binaries to develop and test eccentricity removal. Sturani et al $[119,130]$ perform few-cycle long simulations, exploring a 1-dim line in the 7-dim parameter space. While these simulations have been used to construct a phenomenological waveform-model of precessing $\mathrm{BH}-\mathrm{BH}[119,130]$, the short length of numerical simulations will severely limit the accuracy of this model. Lousto and Zlochower [1] perform 42 simulations to explore black hole kicks with partial spin/orbit alignment. Again, the short length of the simulations (about five orbits) makes them unsuitable for GW data-analysis purposes. Recently, several papers discuss techniques to represent waveforms of precessing binaries by expanding the waveforms in spherical harmonics with respect to a time-dependent frame which is aligned with the instantaneous radiation [131-133] (of which [131] presents a precessing $q=3 \mathrm{BH}-$ BH simulation).

As this short survey illustrates, several groups put their toe into the ocean of precessing binaries, but nobody has seriously braved it yet. The most significant current effort to explore precessing systems is the NR-AR collaboration [122], which aims to construct waveform models for precessing $\mathrm{BH}-\mathrm{BH}$ systems. While an intermediate goal is to revisit the aligned spin case, this collaboration will compute several high-quality precessing waveforms.

\section{4. $B H-B H$ in non-vacuum}

Fully relativistic hydro-dynamics simulations of $\mathrm{BH}-\mathrm{BH}$ in gaseous environment have continued. Bode et al [134] and Bogdanovic et al [135] consider radiatively inefficient accretion flow on merging $\mathrm{BH}-\mathrm{BH}$ binaries, modeled as a $\mathrm{BH}-\mathrm{BH}$ embedded in a cloud of gas with Gaussian density profile centered on the center of mass. Farris et al [136] investigate the $\mathrm{BH}-\mathrm{BH}$ analog of Bondi-accretion, embedding the $\mathrm{BH}-\mathrm{BH}$ in ambient gas of constant density*.

Accretion disks are also under continued investigation. Refs. [138-140] study the behavior of a circumbinary disk after the $\mathrm{BH}-\mathrm{BH}$ merger, when the disk responds to the remnant black hole with reduced mass (due to energy loss through gravitational waves) and with non-zero velocity relative to the center of mass of the accretion disk (due to black hole kicks). More recently, Bode et al. [141] and Farris et al. [142] investigate $\mathrm{BH}-\mathrm{BH}$ binaries with a circumbinary disk.

Two groups are investigating the effects of electro-magnetic fields surrounding BH-BH binaries. This work started with solving the vaccum Maxwell equations coupled to GR by Palenzuela et al $[143,144]$ and Moesta et al. [145]. More recently a force-free treatment of the electro-magnetic fields was presented in Refs. [146-150]. The force-free approximation assumes the presence of a tenuous plasma which shortens out any electric field parallel to the magnetic field lines. Such a plasma is produced by pair-production and forms the basis for the Blandford-Znajek process [151] to extract energy from rotating black holes. Palenzuela et al [146], in particular, demonstrate how a merger of a spinning $\mathrm{BH}-\mathrm{BH}$ can result in a total of three jets: During the inspiral,

* Zanotti et al [137] studied the standard Bondi accretion onto a single black hole with general relativistic radiation-hydrodynamics. 
one jet associated with each $\mathrm{BH}$; and after the merger, a third jet associated with the remnant BH. Moesta et al [150] consider the strength of this beamed emission relative to the uniform emission that also accompanies mergers of $\mathrm{BH}-\mathrm{BH}$ in these cases.

\section{BH-NS \& NS-NS systems}

We now turn to simulations with at least one Neutron star (NS). These simulations have a more varied set of goals than the $\mathrm{BH}-\mathrm{BH}$ simulations, among them:

(i) Compute gravitational waveforms to aid GW detectors. This requires simulations covering many inspiral orbits at high phase-accuracy, a significant challenge for hydro-codes which are typically less accurate than vacuum BH-BH codes.

(ii) Investigate properties of the merger: In BH-NS binaries, does the Neutron star get disrupted? Does an accretion disk form, and how large is it? Is the binary a viable progenitor for a short gamma ray burst?

(iii) Are these mergers a viable source for r-process elements [152]? This requires the formation of unbound ejecta which return material into the interstellar medium.

A variety of physical effects may affect evolution of these binaries and all of these effect must be studied numerically: impact of mass-ratio, effect of equation of state, effect of magnetic fields, effect of nuclear physics, effect of neutrino transport. For BHNS systems, one must also investigate the effect of the $\mathrm{BH}$ spin (both in magnitude and direction). Neutron stars are generally assumed to be very slowly spinning, albeit some recent work approaches simulations of spinning NS $[153,154]$. Several different groups investigate BH-NS and NS-NS systems, with each group generally focusing on a a subset of these physical effects, as detailed in Secs. 3.1 and 3.2.

The numerical techniques for BH-NS and NS-NS combine a solver for the Einstein equations with appropriate solvers for the matter fields. Most commonly used is the BSSNOK evolution system for gravity, combined with high-resolution shock capturing techniques for relativistic hydrodynamics, discretized with adaptive-mesh refinement finite-differences. The SpEC collaboration combines a spectral treatment of the gravity sector (similar to their $\mathrm{BH}-\mathrm{BH}$ simulations) with a treatment of matter on a separate Cartesian finite-difference grid that covers only the region of space in which matter is present and that moves along with the Neutron stars. Some recent papers describing numerical techniques and individual codes are Refs. [155-163]. Initial data for compact object binaries with Neutron stars are described in Refs. [164-169].

Detailed investigations into accuracy of BH-NS and NS-NS simulations are given in Refs. [170-172]. Broadly speaking, preserving low-phase errors during inspiral is difficult and simulations with Neutron stars are less accurate than their $\mathrm{BH}-$ $\mathrm{BH}$ counterparts. No current code is clearly superior; in particular, the advantage of SpEC for vacuum simulations does not carry over and SpEC-hydro-simulations are of comparable accuracy than other codes. Resolving disk-dynamics is another numerically challenging aspect, especially when the disks are long-lived. Finally, MHD simulations require a lower cutoff on the matter density. Because gamma-ray-bursts involve outflows in regions of low Baryon density, the MHD caveats might become important (the IMEX treatment of Palenzuela et al [173] is a recent alternative). 


\section{1. $B H-N S$}

One key objective of the study of BH-NS binaries lies in finding the region of parameter space where the Neutron star is tidally disrupted. In this case, an accretion disk forms and the system is considered a possible candidate for a gamma-ray burst (although disruption may not be necessary for a gamma-ray burst, see $[174,175]$ ). Moreover, the gravitational wave signature changes dramatically at disruption: Once the Neutron star is disrupted and spread into an accretion disk, GW emission is drastically reduced.

Tidal disruption depends primarily on three parameters. (1) The compaction of the Neutron star: more compact stars are harder to disrupt. (2) The mass of the black hole: more massive black holes have weaker tidal forces, reducing the tendency of disruption. (3) The distance between Neutron star and black hole: For spinning black holes, the innermost stable orbit of corotating geodesics moves inward; therefore, for $\mathrm{BH}-\mathrm{NS}$ systems with the $\mathrm{BH}$ spin aligned with the orbital angular momentum, the orbit of the Neutron star will reach a smaller distance, thus increasing the tendency of disruption.

A significant amount of attention has been devoted on determining the parameter space in which accretion disks form and on the properties of the formed accretion disks. Let us summarize some recent work: Shibata et al. [176] investigate polytropic Neutron stars orbiting non-spinning black holes. For mass-ratios $q=M_{\mathrm{BH}} / N_{\mathrm{NS}}=1.5$ and 3 , disruption occurs, but not so for $q=5$. Kyutoku et al. [177] consider BH's with spin aligned or antialigned with the orbital angular momentum with spin magnitudes up to $\chi_{\mathrm{BH}} \equiv S_{\mathrm{BH}} / M_{\mathrm{BH}}^{2}=0.5$. For low mass-ratio $q \leq 3$ the Neutron star disrupts. For $q=5$, disruption occurs only for prograde $\mathrm{BH}$-spin (this work uses a piecewise polytropic equation of state, which is fitted to physical equation of states). The remnant disks have mass of $\sim 0.1 M_{\odot}$ and this work (as the other references mentioned) confirm that indeed NS disruption leads to a cutoff in the gravitational wave spectrum. A related study by Chawla et al. [178] agrees with the results of [177]: Focusing on mass-ratio $q=5$, aligned co-rotating $\mathrm{BH}$ with spin of $\chi_{\mathrm{BH}}=S_{\mathrm{BH}} / M_{\mathrm{BH}}^{2}=0.5$, and a polytropic equation of state, Chawla et al find that an accretion disk forms, with essentially all material gravitationally bound. Ref. [178] also incorporates magnetic fields, and for field-strengths up to $10^{12} \mathrm{G}$, the effect of the magnetic field was found to be marginal.

$\mathrm{BH}-\mathrm{NS}$ binaries with relatively low mass-ratio $q \lesssim 5$ are numerically easier to handle and are interesting because these systems form accretion disks easily. However, population synthesis suggests that black holes are generally more massive [179]. Foucart et al. [171] perform simulations with more massive black holes, $M_{\mathrm{BH}}=10 M_{\odot}$ and mass-ratio $q=7$. For a non-spinning black hole, or moderately corotating black hole, no accretion disk forms at $q=7$. Only at high spins $\chi_{\mathrm{BH}} \gtrsim 0.7$ does an accretion disk develop. These simulations investigate $\mathrm{BHs}$ with spins as large as $\chi_{\mathrm{BH}}=0.9$, the largest to date.

To close our summary on the effect of $\mathrm{BH}$ spin and mass-ratio, we note that all simulations mentioned so far have the $\mathrm{BH}$ spin parallel to the orbital angular momentum. This assumption is removed by Foucart et al. [180] who vary the spin direction of the BH. Specifically, the angle $\theta$ between $\mathrm{BH}$ spin and orbital angular momentum is varied between $0^{\circ}$ and $80^{\circ}$ (for $q=3, \mathrm{BH}$ spin $\chi_{\mathrm{BH}}=0.5$ and a polytropic equation of state). Angles $\theta>40^{\circ}$ result in a reduction in disk-mass by about a factor of 2. For the same mass-ratio, Ref. [180] also investigate aligned BH spins with magnitude $\chi_{\mathrm{BH}}=0.9$. Consistent with expectations, such a high co-rotating $\mathrm{BH}$ spin coupled with the low mass-ratio $q=3$ results in a very large disk with mass 
approaching $\sim 0.4 M_{\mathrm{NS}}$.

A second focal point for recent work was the impact of the equation of state (EOS) of the Neutron star matter. Duez et al. [181] investigate polytropic equations of state with two different polytropic indices $(\Gamma=2$ and $\Gamma=2.75)$, and the Shen EOS with two treatments of the electron fraction. The binary has mass-ratio $q=3$ and an aligned $\mathrm{BH}$ spin $\chi_{\mathrm{BH}}=0.5$. Duez et al find that more compact Neutron stars emit stronger gravitational waves and result in smaller disk-masses. In particular, tidal tails depend on the EOS. Kyotuko et al. [182] investigate piece-wise polytropic equations of state $[183,184]$. They find that less compact Neutron stars tend to disrupt at a larger separation, and that disk-mass (and the characteristic GW cutoff frequency) correlate with compaction of the Neutron star.

Recently, the first MHD simulations of BH-NS binaries were performed. Chawla et al. [178] consider field-strengths of $10^{12} G$ and find no appreciable difference to nonmagnetic binaries, whereas Etienne et al. [185] report that a magnetic field of $10^{17} \mathrm{G}$ results in a increased disk-mass (no evidence for collimated outflows were observed, although [185] points out that higher resolution simulations would be needed, that follow the post-merger dynamics for a longer period of time).

Stephens et al. [186] and East et al. [187] consider hyperbolic encounters of a Neutron star with a black hole (with relative velocity $1000 \mathrm{~km} / \mathrm{sec}$ at large distance). Refs. $[186,187]$ vary the equation of state, impact parameter, and BH spin. By far the biggest effect on the results has the impact parameter: Depending on the periastron distance, the NS can be disrupted in the first approach, periodic mass-transfer can occur, or the NS can pass the BH unharmed.

\section{2. $N S-N S$}

Binary Neutron stars have seen an equal amount of activity lately as BH-NS binaries. The themes are quite similar to $\mathrm{BH}-\mathrm{NS}$, with intense efforts of the research groups to extend the range of included physical effects.

Equation of state effects are exhaustively explored by Hotokezaka et al. [188]. They simulate six different EOS's (parametrized by piecewise polytropic $[183,184]$ ) for three different NS-masses each and classify the results into prompt collapse to a $\mathrm{BH}$, shortlived hypermassive Neutron star, and long-lived hypermassive Neutron stars. Hotokezaka et al report torus masses of up to $0.1 M_{\odot}$ around the newly formed black hole. Sekiguchi et al [189] explore an equation of state with a phase-transition to hyperons. This causes substantial differences in dynamics, observable in gravitational waves.

When a NS-NS merger results in a hypermassive Neutron star (as opposed to prompt collapse), shocks during the merger will heat the remnant NS to high temperatures, and neutrino cooling will become important. Therefore, neutrino cooling must be modeled in order to determine reliably the timescale on which the hypermassive NS cools and collapses to a $\mathrm{BH}$ when the thermal pressure becomes insufficient to support the star. Sekiguchi et al. [190] perform the first simulation of this process, incorporating neutrino cooling with a leakage scheme, and using a finitetemperature Shen EOS. They report the neutrino luminosities for the simulated NSNS mergers, and find indeed that the lifetime of low-mass hypermassive NS depends on the neutrino cooling.

MHD simulations have also been improved. Giacomazzo et al [191] and Rezzolla et al [192] focus on very long simulations of the post-merger accretion disk. While 
Ref. [191] focuses on disk dynamics, Ref. [192] reports evidence of electromagnetic collimation along the rotation axis of the accretion disk. This is the first claim of the "missing link" that connects the post-merger accretion disk with the eventual launching of the jets that power gamma ray bursts. Two other research groups have published simulations of merging magnetized NS-NS systems [193,194], and both these groups have since published improved techniques to handle magnetic fields $[161,162,173,195]$. It will be very interesting to see whether the impressive and important results of Ref. [192] can be confirmed by these groups.

Eccentric NS-NS systems were also studied for the first time. Gold et al. [196] find that eccentric mergers result in larger disks, and that periastron passage leads to excitation of f-modes in the Neutron stars.

Finally, recently work has begun to compare NS-NS inspiral simulations with post-Newtonian approximations, and to fit analytical waveform models to the numerical NS-NS inspiral simulations. Such work is important for gravitational wave data-analysis, because complete, phase-accurate waveforms (ranging from early post-Newtonian inspiral into merger) promise the most accurate data-analysis for gravitational waves from NS-NS. Several NS-NS simulations were performed for these purposes all about 10 orbits long. Bernuzzi et al. [172] performs a comparison with TaylorT4 and finds significant phase-differences (about 1 GW cylce), which cannot be explained by known tidal corrections. Baiotti et al [197,198] investigate TaylorT4 and EOB models. Without free parameters, EOB and TaylorT4 both deviate by about one GW cycle from the numerical NS-NS simulation; in contrast, if the EOB model is amended with one free fitting parameter (parameterizing the strength of higher order tidal effects), the NS-NS simulation can be fitted with an error of only 0.24 radians.

Read et al. [199] and Lackey et al.[200] analyze large sets of NS-NS simulations to determine what information about the equation of state can be extracted from future gravitational wave observations. The best-constrained parameter will be the compactness of the Neutron star.

\section{Conclusion}

The recent progress in simulations of $\mathrm{BH}-\mathrm{BH}, \mathrm{BH}-\mathrm{NS}$ and NS-NS systems is spectacular. What are the challenges going forward?

For $\mathrm{BH}-\mathrm{BH}$, the numerical methods are in good shape. Very challenging simulations were successfully performed as described in Sec. 2.2, pushing large massratio, large spins, and the number of GW-cycles. The open question is whether the numerical techniques can handle a combination of these properties, e.g. high spin $(\gtrsim 0.9)$ and high mass-ratio ( $\gtrsim 4)$, and how well accuracy holds up when the number of GW cycles in the simulation is increased. Besides these unexplored regions of parameter space, the main challenge forward is the systematic exploration of the vast parameter space of possible binaries, at sufficient accuracy and length to allow gravitational wave detectors to reach optimal sensitivity and optimal accuracy in parameter estimation. The computational cost of $\mathrm{BH}-\mathrm{BH}$ simulations, the possibility that the numerical waveforms may have to be much longer than current simulations for optimal parameter estimation, and the sheer size of the parameter space will necessitate compromises, in length of the simulations or in parameter space coverage (or both). The severity of these compromises will only be known after an initial exploration of the precessing parameter space and after first attempts to fit analytical waveform models. The ease of fitting (currently unknown) will determine how many 
simulations are needed.

Efficiency improvements in the numerical codes will furthermore determine how quickly the parameter space can be sampled and how quickly on-demand simulations can be performed (e.g. in response to gravitational wave detectors observing a tentative event). Such efficiency improvements may come from novel computer algorithms like implicit time-stepping $[109,110]$ or from utilizing novel computing paradigms like graphical processing units.

For BH-NS and NS-NS systems, the current frontier is exploration of all relevant physical effects. Given the complexity of the simulations and the varied micro-physics, it is imperative that different groups perform similar simulations to cross-check. Once qualitative features are explored, the field will turn towards quantitative sampling of the parameter space, with systematic and careful calculation of gravitational waveforms.

Numerical and implementation issues of compact object binaries seem a problem of the past, as attested by the stunning progress in numerical relativity. The deeper insight into formulation of the equations, and experience of what works and what does not makes it now possible to consider entirely different numerical algorithms, like discontinuous Galerkin methods [201,202], moving Voronoi meshes [203,204], or novel time-stepping techniques [109].

\section{Acknowledgments}

I thank the organizers of the 9th Eduardo Amaldi conference and the 5th Numerical Relativity-Data Analysis meeting for organizing two extremely stimulating conferences. I thank Matt Duez and Francois Foucart for illuminating discussions, and gratefully acknowledge support from NSERC of Canada, the Canada Chairs Program and the Canadian Institute for Advanced Research.

\section{References}

[1] Lousto C O and Zlochower Y 2011 Phys.Rev.Lett. 107231102 (Preprint 1108.2009)

[2] Cohen M I, Kaplan J D and Scheel M A 2012 Phys.Rev. D85 024031 (Preprint 1110.1668)

[3] Cohen M I, Pfeiffer H P and Scheel M A 2009 Class.Quant.Grav. 26035005 (Preprint 0809.2628)

[4] Ponce M, Lousto C and Zlochower Y 2011 Class.Quant.Grav. 28145027 (Preprint 1008.2761)

[5] Duez M D 2010 Class.Quant.Grav. 27114002 (Preprint 0912.3529)

[6] Shibata M and Taniguchi K 2011 Living Reviews in Relativity 14 URL http://www. livingreviews.org/lrr-2011-6

[7] Abbott B et al. (LIGO Scientific) 2009 Rept. Prog. Phys. 72076901 (Preprint 0711.3041)

[8] Shoemaker D (the Advanced LIGO Team) 2009 Advanced LIGO Reference Design [LIGOM060056]

[9] Harry G M and the LIGO Scientific Collaboration 2010 Class. Quant. Grav. 27084006

[10] Accadia T, Acernese F, Antonucci F, Astone P, Ballardin G et al. 2011 Class. Quant. Grav. 28114002

[11] The Virgo Collaboration 2009 Advanced Virgo Baseline Design [VIR-0027A-09]

[12] Somiya K (for the LCGT Collaboration) 2011 (Preprint 1111.7185)

[13] Centrella J, Baker J G, Kelly B J and van Meter J R 2010 Rev.Mod.Phys. 823069 (Preprint 1010.5260)

[14] McWilliams S T 2011 Class.Quant.Grav. 28134001 (Preprint 1012.2872)

[15] Baumgarte T W and Shapiro S L 2010 Numerical Relativity: Solving Einstein's Equations on the Computer (Cambridge University Press)

[16] Witek H, Zilhao M, Gualtieri L, Cardoso V, Herdeiro C et al. 2010 Phys.Rev. D82 104014 (Preprint 1006.3081)

[17] Lehner L and Pretorius F 2010 Phys.Rev.Lett. 105101102 (Preprint 1006.5960) 
[18] Healy J, Bode T, Haas R, Pazos E, Laguna P et al. 2011 (Preprint 1112.3928)

[19] Paschalidis V, Halataei S M, Shapiro S L and Sawicki I 2011 Class.Quant.Grav. 28085006 (Preprint 1103.0984)

[20] Pretorius F 2005 Phys.Rev.Lett. 95121101 (Preprint gr-qc/0507014)

[21] Campanelli M, Lousto C, Marronetti P and Zlochower Y 2006 Phys.Rev.Lett. 96111101 (Preprint gr-qc/0511048)

[22] Baker J G, Centrella J, Choi D I, Koppitz M and van Meter J 2006 Phys.Rev.Lett. 96111102 (Preprint gr-qc/0511103)

[23] Nakamura T, Oohara K and Kojima Y 1987 Prog. Theor. Phys. Suppl. 90 1-218

[24] Shibata M and Nakamura T 1995 Phys. Rev D52 5428-5444

[25] Baumgarte T W and Shapiro S L 1999 Phys. Rev. D59 024007 (Preprint gr-qc/9810065)

[26] Friedrich H 1985 Commun. Math. Phys. 100 525-543

[27] Pretorius F 2005 Class.Quant.Grav. 22 425-452 (Preprint gr-qc/0407110)

[28] Lindblom L, Scheel M A, Kidder L E, Owen R and Rinne O 2006 Class.Quant.Grav. 23 S447S462 (Preprint gr-qc/0512093)

[29] Sperhake U, Cardoso V, Pretorius F, Berti E and Gonzalez J A 2008 Phys. Rev. Lett. 101 161101 (Preprint 0806.1738)

[30] Pretorius F and Khurana D 2007 Class.Quant.Grav. 24 S83-S108 (Preprint gr-qc/0702084)

[31] Ramazanoglu F M and Pretorius F 2010 Class.Quant.Grav. 27245027 (Preprint 1009.1440)

[32] O'Murchadha N and York J W 1974 Phys.Rev. D10 428-436

[33] Pfeiffer H P and York James W J 2003 Phys.Rev. D67 044022 (Preprint gr-qc/0207095)

[34] Bowen J M and York James W J 1980 Phys.Rev. D21 2047-2056

[35] York James W J 1999 Phys.Rev.Lett. 82 1350-1353 (Preprint gr-qc/9810051)

[36] Brandt S and Bruegmann B 1997 Phys.Rev.Lett. 78 3606-3609 (Preprint gr-qc/9703066)

[37] Caudill M, Cook G B, Grigsby J D and Pfeiffer H P 2006 Phys.Rev. D74 064011 (Preprint gr-qc/0605053)

[38] Cook G B and Pfeiffer H P 2004 Phys.Rev. D70 104016 (Preprint gr-qc/0407078)

[39] Cook G B 2002 Phys.Rev. D65 084003 (Preprint gr-qc/0108076)

[40] Ansorg M, Bruegmann B and Tichy W 2004 Phys.Rev. D70 064011 (Preprint gr-qc/0404056)

[41] Pfeiffer H P, Kidder L E, Scheel M A and Teukolsky S A 2003 Comput.Phys.Commun. 152 253-273 (Preprint gr-qc/0202096)

[42] Husa S, Hannam M, Gonzalez J A, Sperhake U and Bruegmann B 2008 Phys.Rev. D77 044037 (Preprint 0706.0904)

[43] Pfeiffer H P, Brown D A, Kidder L E, Lindblom L, Lovelace G et al. 2007 Class.Quant.Grav. 24 S59-S82 (Preprint gr-qc/0702106)

[44] Buonanno A, Kidder L E, Mroue A H, Pfeiffer H P and Taracchini A 2011 Phys.Rev. D83 104034 (Preprint 1012.1549)

[45] Bona C, Masso J, Seidel E and Stela J 1997 Phys. Rev. D56 3405-3415 (Preprint gr-qc/ 9709016)

[46] Alcubierre M et al. 2003 Phys. Rev. D67 084023 (Preprint gr-qc/0206072)

[47] van Meter J R, Baker J G, Koppitz M and Choi D I 2006 Phys.Rev. D73 124011 (Preprint gr-qc/0605030)

[48] Szilagyi B, Lindblom L and Scheel M A 2009 Phys.Rev. D80 124010 (Preprint 0909.3557)

[49] Scheel M A, Pfeiffer H P, Lindblom L, Kidder L E, Rinne O et al. 2006 Phys.Rev. D74 104006 (Preprint gr-qc/0607056)

[50] Rinne O, Lindblom L and Scheel M A 2007 Class.Quant.Grav. 24 4053-4078 (Preprint 0704.0782)

[51] Husa S, Gonzalez J A, Hannam M, Bruegmann B and Sperhake U 2008 Class.Quant.Grav. 25 105006 (Preprint 0706.0740)

[52] Zlochower Y, Baker J, Campanelli M and Lousto C 2005 Phys.Rev. D72 024021 (Preprint gr-qc/0505055)

[53] Brown J, Sarbach O, Schnetter E, Tiglio M, Diener P et al. 2007 Phys.Rev. D76 081503 (Preprint 0707.3101)

[54] Brown J, Diener P, Sarbach O, Schnetter E and Tiglio M 2009 Phys.Rev. D79 044023 (Preprint 0809.3533)

[55] Etienne Z B, Faber J A, Liu Y T, Shapiro S L and Baumgarte T W 2007 Phys.Rev. D76 101503 (Preprint 0707.2083)

[56] Lovelace G, Boyle M, Scheel M A and Szilagyi B 2012 Class.Quant.Grav. 29045003 (Preprint 1110.2229)

[57] Hannam M, Husa S and Murchadha N O 2009 Phys.Rev. D80 124007 (Preprint 0908.1063)

[58] Goodale T, Allen G, Lanfermann G, Massó J, Radke T, Seidel E and Shalf J 2003 Vector 
and Parallel Processing - VECPAR'2002, 5th International Conference, Lecture Notes in Computer Science (Berlin: Springer) pp 197-227

[59] Cactus computational toolkit URL http://www.cactuscode.org/

[60] Loffler F, Faber J, Bentivegna E, Bode T, Diener P et al. 2011 (Preprint 1111.3344)

[61] Schnetter E, Hawley S H and Hawke I 2004 Class.Quant.Grav. 21 1465-1488 (Preprint gr-qc/0310042)

[62] Schnetter E Carpet: A mesh refinement driver for cactus URL http://www.carpetcode.org/

[63] Thornburg J 2004 Class.Quant.Grav. 21 743-766 (Preprint gr-qc/0306056)

[64] Ajith P, Boyle M, Brown D A, Brugmann B, Buchman L T et al. 2012 (Preprint 1201.5319)

[65] http://www.black-holes.org/SpEC.html

[66] Owen R, Brink J, Chen Y, Kaplan J D, Lovelace G et al. 2011 Phys.Rev.Lett. 106151101 (Preprint 1012.4869)

[67] Nichols D A, Owen R, Zhang F, Zimmerman A, Brink J et al. 2011 Phys.Rev. D84 124014 (Preprint 1108.5486)

[68] Muller D, Grigsby J and Bruegmann B 2010 Phys.Rev. D82 064004 (Preprint 1003.4681)

[69] Schnetter E 2010 Class.Quant.Grav. 27167001 (Preprint 1003.0859)

[70] Alic D, Rezzolla L, Hinder I and Mosta P 2010 Class.Quant.Grav. 27245023 (Preprint 1008.2212)

[71] Chu T, Pfeiffer H P and Scheel M A 2009 Phys.Rev. D80 124051 (Preprint 0909.1313)

[72] Sperhake U, Cardoso V, Ott C D, Schnetter E and Witek H 2011 Phys.Rev. D84 084038 (Preprint 1105.5391)

[73] Lousto C O and Zlochower Y 2011 Phys.Rev.Lett. 106041101 (Preprint 1009.0292)

[74] Nakano H, Zlochower Y, Lousto C O and Campanelli M 2011 Phys. Rev. D84 124006 (Preprint 1108.4421)

[75] Lousto C O, Nakano H, Zlochower Y and Campanelli M 2010 Phys. Rev. D82 104057 (Preprint 1008.4360)

[76] Lousto C O, Nakano H, Zlochower Y and Campanelli M 2010 Phys. Rev. Lett. 104211101 (Preprint 1001.2316)

[77] Dain S, Lousto C O and Zlochower Y 2008 Phys.Rev. D78 024039 (Preprint 0803.0351)

[78] Lovelace G, Owen R, Pfeiffer H P and Chu T 2008 Phys.Rev. D78 084017 (Preprint 0805.4192)

[79] Lovelace G, Scheel M and Szilagyi B 2011 Phys.Rev. D83 024010 (Preprint 1010.2777)

[80] Le Tiec A, Mroue A H, Barack L, Buonanno A, Pfeiffer H P et al. 2011 Phys.Rev.Lett. 107 141101 (Preprint 1106.3278)

[81] Healy J, Herrmann F, Hinder I, Shoemaker D M, Laguna P et al. 2009 Phys.Rev.Lett. 102 041101 (Preprint 0807.3292)

[82] Campanelli M, Lousto C O, Zlochower Y and Merritt D 2007 Astrophys.J. 659 L5-L8 (Preprint gr-qc/0701164)

[83] Gonzalez J, Hannam M, Sperhake U, Bruegmann B and Husa S 2007 Phys.Rev.Lett. 98231101 (Preprint gr-qc/0702052)

[84] Campanelli M, Lousto C O, Zlochower Y and Merritt D 2007 Phys.Rev.Lett. 98231102 (Preprint gr-qc/0702133)

[85] Peters P 1964 Phys.Rev. 136 B1224-B1232

[86] Peters P and Mathews J 1963 Phys.Rev. 131 435-439

[87] Walther B, Bruegmann B and Mueller D 2009 Phys.Rev. D79 124040 (Preprint 0901.0993)

[88] Boyle M, Brown D A, Kidder L E, Mroue A H, Pfeiffer H P et al. 2007 Phys.Rev. D76 124038 (Preprint 0710.0158)

[89] Tichy W and Marronetti P 2011 Phys.Rev. D83 024012 (Preprint 1010.2936)

[90] Purrer M, Husa S and Hannam M 2012 (Preprint 1203.4258)

[91] Babiuc M, Bishop N, Szilagyi B and Winicour J 2009 Phys.Rev. D79 084011 (Preprint 0808.0861)

[92] Reisswig C, Bishop N, Pollney D and Szilagyi B 2009 Phys.Rev.Lett. 103221101 (Preprint 0907.2637)

[93] Babiuc M, Szilagyi B, Winicour J and Zlochower Y 2011 Phys.Rev. D84 044057 (Preprint 1011.4223)

[94] Babiuc M, Winicour J and Zlochower Y 2011 Class.Quant.Grav. 28134006 (Preprint 1106. 4841)

[95] Boyle M and Mroue A H 2009 Phys.Rev. D80 124045 (Preprint 0905.3177)

[96] http://einsteintoolkit.org

[97] Bishop N, Pollney D and Reisswig C 2011 Class.Quant.Grav. 28155019 (Preprint 1101.5492)

[98] Owen R 2010 Phys.Rev. D81 124042 (Preprint 1004.3768)

[99] Owen R 2009 Phys.Rev. D80 084012 (Preprint 0907.0280) 
[100] Campanelli M, Lousto C O and Zlochower Y 2009 Phys.Rev. D79 084012 (Preprint 0811.3006 )

[101] Hinder I, Wardell B and Bentivegna E 2011 Phys.Rev. D84 024036 (Preprint 1105.0781)

[102] Pollney D, Reisswig C, Dorband N, Schnetter E and Diener P 2009 Phys.Rev. D80 121502 (Preprint 0910.3656)

[103] https://www.ninja-project.org

[104] Alic D, Bona-Casas C, Bona C, Rezzolla L and Palenzuela C 2011 (Preprint 1106.2254)

[105] Witek H, Hilditch D and Sperhake U 2011 Phys.Rev. D83 104041 (Preprint 1011.4407)

[106] Bona C, Bona-Casas C and Palenzuela C 2010 Phys.Rev. D82 124010 (Preprint 1008.0747)

[107] Bruegmann B 2011 (Preprint 1104.3408)

[108] Mroué A H 2010 Binary black hole simulations using CUDA NVIDIA GPU Technology Conference

[109] Lau S R, Lovelace G and Pfeiffer H P 2011 Phys.Rev. D84 084023 (Preprint 1105.3922)

[110] Lau S R, Pfeiffer H P and Hesthaven J S 2008 Comput.Phys.Commun. (Preprint 0808.2597)

[111] Hennig J and Ansorg M 2009 J.Hyperbol.Diff.Equat. 6161 (Preprint 0801.1455)

[112] Ohme F 2011 (Preprint 1111.3737)

[113] Pan Y, Buonanno A, Baker J G, Centrella J, Kelly B J et al. 2008 Phys.Rev. D77 024014 (Preprint 0704.1964)

[114] Buonanno A, Pan Y, Baker J G, Centrella J, Kelly B J et al. 2007 Phys.Rev. D76 104049 (Preprint 0706.3732)

[115] Damour T, Nagar A, Hannam M, Husa S and Bruegmann B 2008 Phys.Rev. D78 044039 (Preprint 0803.3162)

[116] Ajith P, Hannam M, Husa S, Chen Y, Bruegmann B et al. 2011 Phys.Rev.Lett. 106241101 (Preprint 0909.2867)

[117] Pan Y, Buonanno A, Buchman L T, Chu T, Kidder L E et al. 2010 Phys.Rev. D81 084041 (Preprint 0912.3466)

[118] Santamaria L, Ohme F, Ajith P, Bruegmann B, Dorband N et al. 2010 Phys.Rev. D82 064016 (Preprint 1005.3306)

[119] Sturani R, Fischetti S, Cadonati L, Guidi G, Healy J et al. 2010 (Preprint 1012.5172)

[120] Pan Y, Buonanno A, Boyle M, Buchman L T, Kidder L E et al. 2011 Phys.Rev. D84 124052 26 pages, 25 figures, published Phys. Rev. D version (Preprint 1106.1021)

[121] Aylott B, Baker J G, Boggs W D, Boyle M, Brady P R et al. 2009 Class.Quant.Grav. 26 165008 (Preprint 0901.4399)

[122] https://www.ninja-project.org/doku.php?id=nrar:home

[123] Blanchet L 2006 Living Rev.Rel. 94

[124] Ohme F, Hannam M and Husa S 2011 Phys.Rev. D84 064029 (Preprint 1107.0996)

[125] Hannam M, Husa S, Ohme F and Ajith P 2010 Phys.Rev. D82 124052 (Preprint 1008.2961)

[126] Damour T, Nagar A and Trias M 2011 Phys.Rev. D83 024006 (Preprint 1009.5998)

[127] Boyle M 2011 Phys.Rev. D84 064013 (Preprint 1103.5088)

[128] MacDonald I, Nissanke S, Pfeiffer H P and Pfeiffer H P 2011 Class.Quant.Grav. 28134002 (Preprint 1102.5128)

[129] Campanelli M, Lousto C O, Nakano H and Zlochower Y 2009 Phys. Rev. D79 084010 (Preprint 0808.0713)

[130] Sturani R, Fischetti S, Cadonati L, Guidi G, Healy J et al. 2010 J.Phys.Conf.Ser. 243012007 (Preprint 1005.0551)

[131] Schmidt P, Hannam M, Husa S and Ajith P 2011 Phys.Rev. D84 024046 (Preprint 1012.2879)

[132] O'Shaughnessy R, Vaishnav B, Healy J, Meeks Z and Shoemaker D 2011 Phys.Rev. D84 124002 (Preprint 1109.5224)

[133] Boyle M, Owen R and Pfeiffer H P 2011 Phys.Rev. D84 124011 (Preprint 1110.2965)

[134] Bode T, Haas R, Bogdanovic T, Laguna P and Shoemaker D 2010 Astrophys.J. 715 1117-1131 (Preprint 0912.0087)

[135] Bogdanovic T, Bode T, Haas R, Laguna P and Shoemaker D 2011 Class.Quant.Grav. 28094020 (Preprint 1010.2496)

[136] Farris B D, Liu Y T and Shapiro S L 2010 Phys.Rev. D81 084008 (Preprint 0912.2096)

[137] Zanotti O, Roedig C, Rezzolla L and Del Zanna L 2011 Mon.Not.Roy.Astron.Soc. 417 28992915 (Preprint 1105.5615)

[138] Anderson M, Lehner L, Megevand M and Neilsen D 2010 Phys.Rev. D81 044004 (Preprint 0910.4969

[139] Megevand M, Anderson M, Frank J, Hirschmann E W, Lehner L et al. 2009 Phys.Rev. D80 024012 (Preprint 0905.3390)

[140] Zanotti O, Rezzolla L, Del Zanna L and Palenzuela C 2010 Astron.Astrophys. 523 A8 (Preprint 1002.4185 
[141] Bode T, Bogdanovic T, Haas R, Healy J, Laguna P et al. 2012 Astrophys.J. 74445 (Preprint $1101.4684)$

[142] Farris B D, Liu Y T and Shapiro S L 2011 Phys.Rev. D84 024024 (Preprint 1105.2821)

[143] Palenzuela C, Lehner L and Yoshida S 2010 Phys.Rev. D81 084007 (Preprint 0911.3889)

[144] Palenzuela C, Anderson M, Lehner L, Liebling S L and Neilsen D 2009 Phys.Rev.Lett. 103 081101 (Preprint 0905.1121)

[145] Mosta P, Palenzuela C, Rezzolla L, Lehner L, Yoshida S et al. 2010 Phys.Rev. D81 064017 (Preprint 0912.2330)

[146] Palenzuela C, Lehner L and Liebling S L 2010 Science 329927 (Preprint 1005.1067)

[147] Palenzuela C, Garrett T, Lehner L and Liebling S L 2010 Phys.Rev. D82 044045 (Preprint 1007.1198)

[148] Neilsen D, Lehner L, Palenzuela C, Hirschmann E W, Liebling S L et al. 2011 Proc.Nat.Acad.Sci. 108 12641-12646 (Preprint 1012.5661)

[149] Palenzuela C, Bona C, Lehner L and Reula O 2011 Class.Quant.Grav. 28134007 (Preprint 1102.3663)

[150] Moesta P, Alic D, Rezzolla L, Zanotti O and Palenzuela C 2011 (Preprint 1109.1177)

[151] Blandford R and Znajek R 1977 Mon.Not.Roy.Astron.Soc. 179 433-456

[152] Freiburghaus C, Rosswog S and Thielemann F K 1999 Astrophys. J. Lett. 525 L121-L124

[153] Baumgarte T W and Shapiro S L 2009 Phys.Rev. D80 064009 (Preprint 0909.0952)

[154] Tichy W 2011 Phys.Rev. D84 024041 (Preprint 1107.1440)

[155] Yamamoto T, Shibata M and Taniguchi K 2008 Phys.Rev. D78 064054 (Preprint 0806.4007)

[156] Giacomazzo B and Rezzolla L 2007 Class.Quant. Grav. 24 S235-S258 (Preprint gr-qc/0701109)

[157] Baiotti L, Shibata M and Yamamoto T 2010 Phys.Rev. D82 064015 (Preprint 1007.1754)

[158] Duez M D et al. 2008 Phys. Rev. D78 104015 (Preprint 0809.0002)

[159] East W E, Pretorius F and Stephens B C 2011 (Preprint 1112.3094)

[160] Thierfelder M, Bernuzzi S and Bruegmann B 2011 Phys.Rev. D84 044012 (Preprint 1104.4751)

[161] Etienne Z B, Paschalidis V, Liu Y T and Shapiro S L 2012 Phys.Rev. D85 024013 (Preprint 1110.4633

[162] Etienne Z B, Liu Y T and Shapiro S L 2010 Phys.Rev. D82 084031 (Preprint 1007.2848)

[163] Anderson M, Hirschmann E, Liebling S L and Neilsen D 2006 Class.Quant.Grav. 23 6503-6524 (Preprint gr-qc/0605102)

[164] Uryu K and Tsokaros A 2012 Phys.Rev. D85 064014 revised version with a new title, 29 pages (Preprint 1108.3065)

[165] Kyutoku K, Shibata M and Taniguchi K 2009 Phys. Rev. D79 124018 (Preprint 0906.0889)

[166] Uryu K, Limousin F, Friedman J L, Gourgoulhon E and Shibata M 2009 Phys.Rev. D80 124004 (Preprint 0908.0579)

[167] Grandclement P 2006 Phys.Rev. D74 124002 (Preprint gr-qc/0609044)

[168] Taniguchi K, Baumgarte T W, Faber J A and Shapiro S L 2007 Phys.Rev. D75 084005 (Preprint gr-qc/0701110)

[169] Foucart F, Kidder L E, Pfeiffer H P and Teukolsky S A 2008 Phys. Rev. D77 124051 (Preprint 0804.3787)

[170] Baiotti L, Giacomazzo B and Rezzolla L 2009 Class.Quant.Grav. 26114005 (Preprint $0901.4955)$

[171] Foucart F, Duez M D, Kidder L E, Scheel M A, Szilagyi B et al. 2012 Phys.Rev. D85 044015 11 pages, 11 figures - Updated to match published version (Preprint 1111.1677)

[172] Bernuzzi S, Thierfelder M and Bruegmann B 2011 (Preprint 1109.3611)

[173] Palenzuela C, Lehner L, Reula O and Rezzolla L 2009 Mon. Not. Roy. Astron. Soc. 394 17271740 (Preprint 0810.1838)

[174] Hansen B M and Lyutikov M 2001 Mon.Not.Roy.Astron.Soc. 322695 (Preprint astro-ph/ 0003218)

[175] McWilliams S T and Levin J 2011 Astrophys.J. 74290 (Preprint 1101.1969)

[176] Shibata M, Kyutoku K, Yamamoto T and Taniguchi K 2009 Phys.Rev. D79 044030 (Preprint 0902.0416)

[177] Kyutoku K, Okawa H, Shibata M and Taniguchi K 2011 Phys. Rev. D84 064018 (Preprint 1108.1189)

[178] Chawla S et al. 2010 Phys. Rev. Lett. 105111101 (Preprint 1006.2839)

[179] Belczynski K, Dominik M, Bulik T, O'Shaughnessy R, Fryer C et al. 2010 (Preprint 1004.0386)

[180] Foucart F, Duez M D, Kidder L E and Teukolsky S A 2011 Phys. Rev. D83 024005 (Preprint $1007.4203)$

[181] Duez M D, Foucart F, Kidder L E, Ott C D and Teukolsky S A 2010 Class. Quant. Grav. 27 114106 (Preprint 0912.3528) 
[182] Kyutoku K, Shibata M and Taniguchi K 2010 Phys. Rev. D82 044049 (Preprint 1008.1460)

[183] Read J S, Lackey B D, Owen B J and Friedman J L 2009 Phys.Rev. D79 124032 (Preprint $0812.2163)$

[184] Ozel F and Psaltis D 2009 Phys.Rev. D80 103003 (Preprint 0905.1959)

[185] Etienne Z B, Liu Y T, Paschalidis V and Shapiro S L 2011 (Preprint 1112.0568)

[186] Stephens B C, East W E and Pretorius F 2011 Astrophys.J. 737 L5 (Preprint 1105.3175)

[187] East W E, Pretorius F and Stephens B C 2011 (Preprint 1111.3055)

[188] Hotokezaka K, Kyutoku K, Okawa H, Shibata M and Kiuchi K 2011 Phys.Rev. D83 124008 (Preprint 1105.4370)

[189] Sekiguchi Y, Kiuchi K, Kyutoku K and Shibata M 2011 Phys.Rev.Lett. 107211101 (Preprint 1110.4442)

[190] Sekiguchi Y, Kiuchi K, Kyutoku K and Shibata M 2011 Phys.Rev.Lett. 107051102 (Preprint 1105.2125)

[191] Giacomazzo B, Rezzolla L and Baiotti L 2011 Phys.Rev. D83 044014 (Preprint 1009.2468)

[192] Rezzolla L, Giacomazzo B, Baiotti L, Granot J, Kouveliotou C et al. 2011 Astrophys.J. 732 L6 (Preprint 1101.4298)

[193] Liu Y T, Shapiro S L, Etienne Z B and Taniguchi K 2008 Phys. Rev. D78 024012 (Preprint 0803.4193)

[194] Anderson M et al. 2008 Phys. Rev. Lett. 100191101 (Preprint 0801.4387)

[195] Liebling S L, Lehner L, Neilsen D and Palenzuela C 2010 Phys. Rev. D81 124023 (Preprint 1001.0575)

[196] Gold R, Bernuzzi S, Thierfelder M, Bruegmann B and Pretorius F 2011 (Preprint 1109.5128)

[197] Baiotti L, Damour T, Giacomazzo B, Nagar A and Rezzolla L 2010 Phys.Rev.Lett. 105261101 (Preprint 1009.0521)

[198] Baiotti L, Damour T, Giacomazzo B, Nagar A and Rezzolla L 2011 Phys.Rev. D84 024017 (Preprint 1103.3874)

[199] Read J S, Markakis C, Shibata M, Uryu K, Creighton J D et al. 2009 Phys.Rev. D79 124033 (Preprint 0901.3258)

[200] Lackey B D, Kyutoku K, Shibata M, Brady P R and Friedman J L 2012 Phys.Rev. D85 044061 (Preprint 1109.3402)

[201] Hesthaven J S and Warburton T 2008 Nodal Discontinuous Galerkin Methods: Algorithms, Analysis, and Applications (Springer)

[202] Radice D and Rezzolla L 2011 Phys.Rev. D84 024010 (Preprint 1103.2426)

[203] Duffell P C and MacFadyen A I 2011 Astrophys.J.Suppl. 19715 (Preprint 1104.3562)

[204] Springel V 2011 (Preprint 1109.2218) 